\title{
Dual-catalytic transition metal systems for functionalization of unreactive sites of molecules
}

\author{
Dawid Lichosyt ${ }^{1}$, Yang Zhang $^{1}$, Karolina Hurej ${ }^{1}$, and Paweł Dydio ${ }^{1 *}$
}

Catalytic reactions occur readily at sites of starting materials that are both innately reactive and sterically accessible or that are predisposed by a functional group amenable to direct a catalyst. However, selective reactions at unbiased sites of substrates remain challenging and typically require additional pre-activation steps or the use of highly reactive reagents. Herein, we report dual-catalytic transition metal systems that merge a reversible activation cycle with a functionalization cycle, together enabling functionalization of substrates at their inherently unreactive sites. By engaging the $\mathrm{Ru}$ - or Fe-catalyzed equilibrium between an alcohol and an aldehyde, Pd-catalyzed $\beta$-arylation of aliphatic alcohols and Rh-catalyzed $\boldsymbol{\gamma}$-hydroarylation of allylic alcohols were developed. The mild conditions, functional group tolerance and broad scope of the methodologies (81 examples) demonstrate the synthetic applicability of the dual-catalytic systems. In a broader context, this work highlights the potential of the multi-catalytic approach to address challenging transformations to circumvent the multi-step procedures and the use of highly reactive reagents in organic synthesis.

By exploiting a set of elementary steps occurring at the metal centers, transition metal (TM) catalysts furnish various reaction pathways, enabling myriads of transformations that greatly empower organic synthesis. ${ }^{1}$ Conventionally, a TM catalyst targets a site that is both innately reactive and sterically accessible ${ }^{2}$ or that is predisposed by the neighboring functional group that directs the catalyst. ${ }^{3}$ Therefore, by careful crafting the microenvironment around the catalytically active metal, a catalyst gains the potential to modulate the inherent reactivity of a substrate to undergo a reaction with an altered selectivity pattern. However, the selective functionalization of any site of a substrate that is electronically and sterically unbiased or undirected by a functional group is challenging and remains largely inaccessible (Fig. 1a). ${ }^{2,4,5}$ Hence, the functionalization of a substrate at such site requires its pre-activation, either by the functional group interconversions that modify the inherent reactivity pattern, converting a target unreactive site into a reactive one, or by the installation of a new functional group amenable to serve as a directing group (Fig. 1b). ${ }^{3}$ Although, such multistep procedure enables the overall target transformation, the approach lengthens synthesis and consumes additional resources, resulting in poor economy and limited utility of the process. Thus, the synthetic methodologies enabling for direct and selective functionalization of typically unreactive sites are highly desired. ${ }^{4,5}$

To simplify the multistep procedures encumbered with the use of stoichiometric resources for the substrate pre-activation, the elegant strategies utilizing reversible reactions have been developed. In one approach, by exploiting dynamic covalent bonding, the steps of the installation and removal of 
a (transient) directing group occur in situ, along with the actual TM catalyzed functionalization step (Fig. 1c). ${ }^{6}$ This approach enables for a series of selective $\mathrm{C}-\mathrm{H}$ bond functionalization reactions to occur in one pot and with the use of only catalytic amounts of activating directing groups. ${ }^{7,8}$ However, the strategy is intrinsically limited to the substrates amenable to form dynamic covalent bonding. In another approach, the structure of a substrate is modulated by a reversible TM catalyzed functional group interconversion (Fig. 1d). ${ }^{9}$ The temporarily formed intermediate possessing an altered reactivity profile undergoes a direct functionalization reaction, followed with subsequent steps involving the initial catalyst to complete the sequence (Fig. 1d). For instance, the alcohols react reversible with some TM complexes, forming reactive aldehyde/ketone intermediates and TM hydride complexes. ${ }^{10,11,12}$ Such reactivity, coined 'hydrogen borrowing', was employed in cascade sequences of reactions to enable amination of alcohols, ${ }^{13-15}$ alkylation reactions ${ }^{9,16}$ and others. ${ }^{17-21}$ The functionalization can be additionally assisted by an organocatalyst to control stereoselectivity of the reaction, ${ }^{21,22}$ though the reactivity still remains limited to the scope of uncatalyzed reactions of the carbonyl intermediate. ${ }^{20}$ Overall, the approaches are generally limited to the use of either an intrinsically reversible uncatalyzed reaction for the activation together with a TM catalyzed functionalization reaction (Fig. 1c), or a TM catalyzed reversible reaction for the activation together with an intrinsic uncatalyzed functionalization reaction (Fig. 1d). These limitations restrict the range of accessible direct transformations that can occur at the typically unreactive sites of substrates.

Inspired by the working principles of the dual-catalytic system for alkane metathesis occurring through the sequence of dehydrogenation, olefin metathesis and hydrogenation, ${ }^{23,24}$ we envisioned that at the intersection of these strategies for the substrate functionalization lies a third powerful approach in which a TM catalyzed reversible reaction for the activation is merged with an orthogonal TM catalyzed functionalization reaction (Fig. 1e). We envisioned that by the combination of these two orthogonal TM catalyzed processes, the reactivity of the intermediate from the functionalization cycle is transposed on the reactivity of the substrate through the activation cycle, together creating a new space of reactivity for the substrate. Here we report such TM dual-catalytic systems, in which the reversible oxidation of an alcohol group by catalytic $\mathrm{Ru}$ - and $\mathrm{Fe}$-complexes enables arylation of the $\beta$ $\mathrm{C}-\mathrm{H}$ bond of aliphatic alcohols by a Pd-catalyst, and $\gamma$-regio- and enantioselective hydroarylation of the $\mathrm{C}=\mathrm{C}$ double bond of allylic alcohols by a Rh-catalyst. Due to the mild reagents and conditions of both the reversible reaction and the functionalization reaction, the devised methodologies are general and compatible with a broad scope of substrates, including natural product-like molecules (81 examples). Given the (microscopic) reversibility of various TM catalyzed processes and an ever increasing number of TM catalyzed functionalization reactions, along with the straightforward design, we anticipate that the presented TM dual-catalytic systems will set the stage to give access to a large repertoire of previously unattainable chemical transformations. 


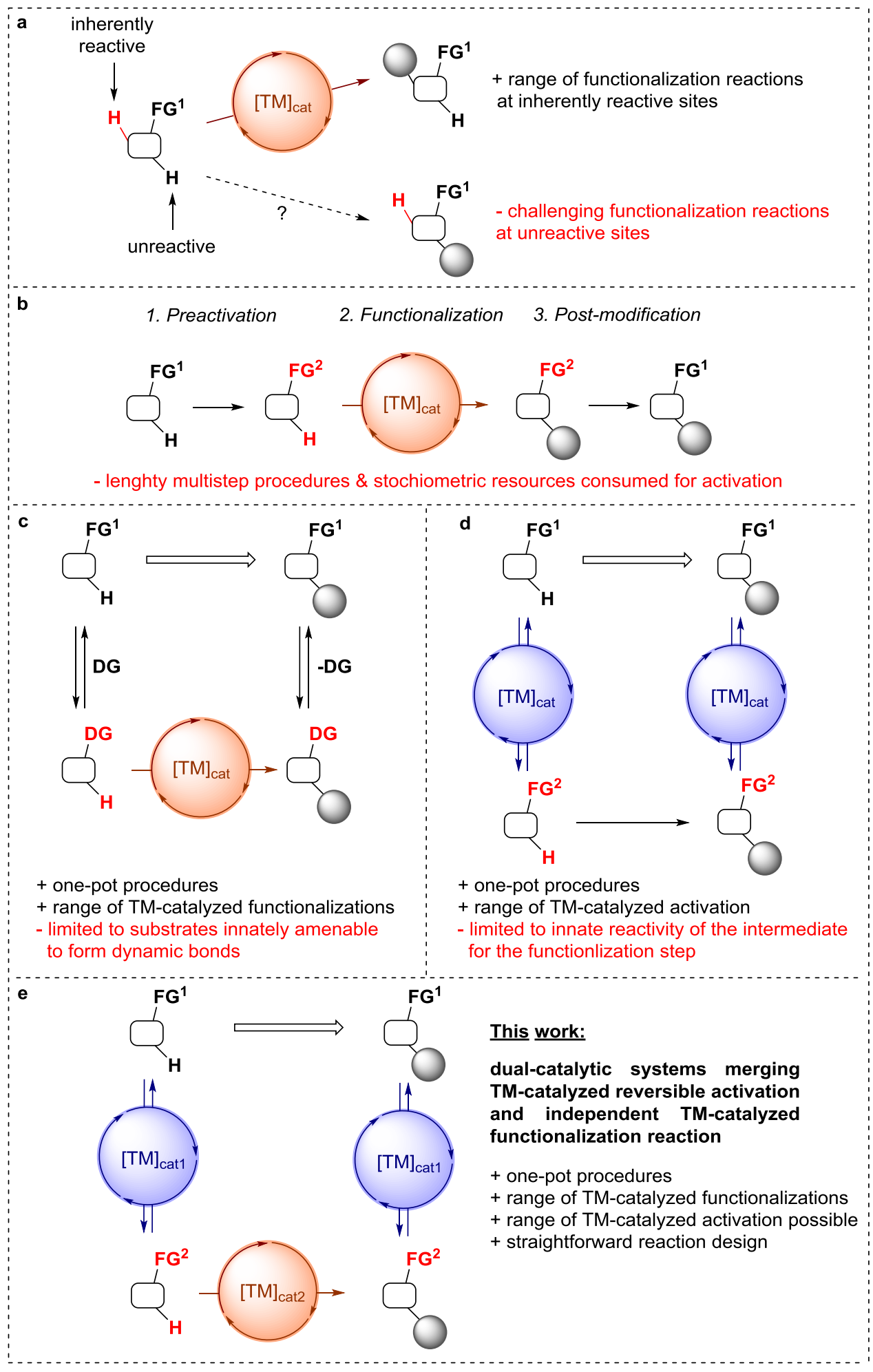

Figure 1 | Transition metal (TM) catalyzed functionalization of starting materials. a, Unreactive versus reactive sites of molecules. b, Multistep strategy for functionalization of unreactive sites of molecules. c, Onepot approach using transient directing group strategy. d, One-pot approach using TM-catalyzed reversible functional group interconversion (e.g. "hydrogen borrowing"). e, Envisioned dual-catalytic strategy involving TM catalyzed reversible functional group interconversion for the transient substrate activation and TM catalyzed functionalization reaction. 


\section{Results and discussion}

To exploit the concept of the TM dual-catalytic systems, we focused on aliphatic alcohols, prevalent organic molecules containing a number of $\mathrm{C}-\mathrm{H}$ bonds that are notorious for their difficulty for functionalization. ${ }^{25}$ In contrast, upon oxidation of an alcohol to its carbonyl derivative - that is, to the aldehyde or the ketone - some originally unreactive $\mathrm{C}-\mathrm{H}$ bonds become pre-activated for $\mathrm{C}-\mathrm{H}$ bond functionalization reactions. ${ }^{26-30}$ For instance, the initially unreactive $\beta$-C-H bonds become acidic and can undergo various TM catalyzed functionalization reactions, ${ }^{26,27}$ including arylation with aryl halides ArX in the presence of a Pd-catalyst and a base (Fig. 2a). To exploit this distinct difference in reactivity, we sought to discover a methodology for the $\beta$-arylation of alcohols enabled by a TM dualcatalytic system combining the reversible hydrogen borrowing reactivity of alcohols and the arylation of aldehydes (Fig. 2a).

In such dual-catalytic system, one transition metal catalyst [TM] reversibly oxidizes the alcohol 1 to the aldehyde $\mathbf{2}$ (Fig. $2 \mathrm{~b}$ ). This temporary change modulates the reactivity of the inert $\beta$-C-H bonds of $\mathbf{1}$ and enables the subsequent reaction. Transient aldehyde $\mathbf{2}$ undergoes arylation with aryl halide $\mathbf{3}$ in the presence of the Pd-catalyst [Pd]. Finally, the aryl aldehyde intermediate 4 reacts with the metal hydride species $[\mathrm{TM}] \mathrm{H}_{2}$ that was generated during the activation step of the alcohol. The final product, that is $\beta$-aryl alcohol 5, is formed, and the complex [TM] is regenerated. The latter can then start a new catalytic turnover with another molecule of $\mathbf{1}$. The catalyst [Pd] alone is unable to catalyze direct arylation of alcohol 1 to give product 5. Although, as we hypothesized, the inclusion of the ancillary $[\mathrm{TM}]$ enables the overall reaction by affording the transient presence of aldehydes $\mathbf{2}$ and $\mathbf{4}$.

We commenced our studies by assessing the reactivity and the compatibility of a series of known Ru-, Ir- and Rh-complexes for hydrogen borrowing reactivity and a series of known Pdcatalysts for aldehyde arylation (Supplementary Table 1). We found that 1-octanol (1a) and bromobenzene (3a) react to form 2-phenyl-1-octanol (5a) in $61 \%$ yield at $80{ }^{\circ} \mathrm{C}$ in $36 \mathrm{~h}$ in dioxane in the presence of $\mathrm{RuCl}_{2}\left(\mathrm{PPh}_{3}\right)_{3}$, the Pd-precatalyst dimer developed by Buchwald (Pd-G3), ${ }^{31}$ dppf, and $\mathrm{Cs}_{2} \mathrm{CO}_{3}$ (Fig. 2c). Under these conditions, the reaction mixture contained $16 \%$ of the aryl aldehyde $\mathbf{4 a}$, as the main side product. However, $\mathbf{4 a}$ is readily converted to target alcohol $\mathbf{5 a}$ upon addition of isopropanol as a reductant, increasing the yield of $\mathbf{5 a}$ to $76 \%$. In general, the results demonstrate that the TM dual-catalytic system exploiting the reversible activation of a substrate is attainable and specific catalysts and conditions suppress competing reactions, favoring the target transformation. 


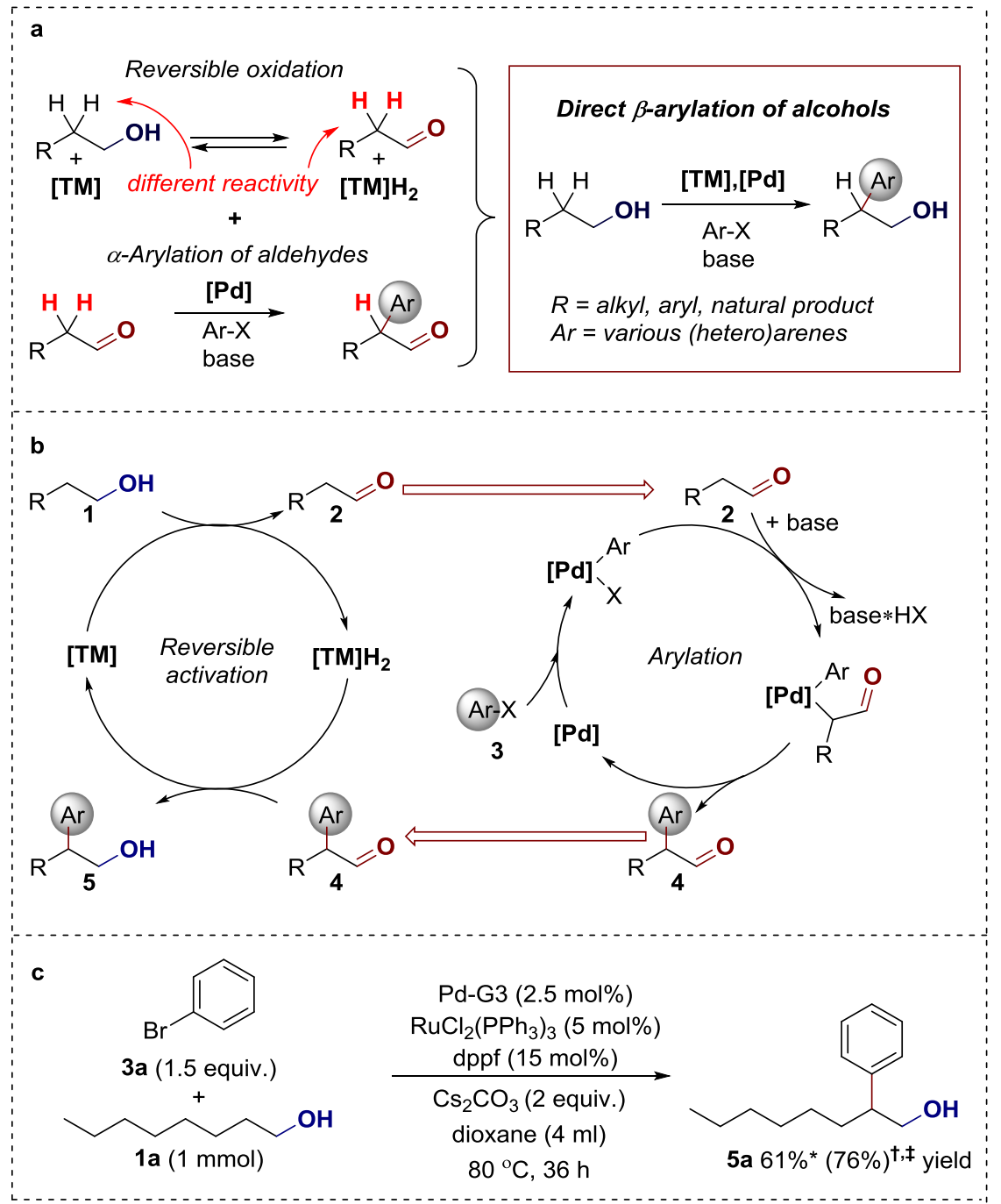

Figure 2 | Reaction design and development. a, Design of the dual-catalytic system: hydrogen borrowing reaction for alcohols and its influence on the reactivity of neighboring $\mathrm{C}-\mathrm{H}$ bonds; $\mathrm{Pd}$-catalyzed arylation of aldehydes; and their proposed combination to enable direct $\beta$-arylation of alcohols. b. Proposed reaction mechanism. c, Model reaction and identified reaction conditions. *Yield determined by NMR using 1,3,5trimetoxybenzene as an internal standard. $\uparrow$ Yield upon addition of isopropanol $(4 \mathrm{ml})$ and additional incubation at $80{ }^{\circ} \mathrm{C}$ for $6 \mathrm{~h}$. $\$$ Isolated yield of $62 \%$. Pd-G3 = the third generation (G3) Buchwald precatalyst dimer; ${ }^{31} \mathrm{dppf}$ $=1,1^{\prime}$-ferrocenediyl-bis(diphenylphosphine).

We conducted the control experiments and the mechanistic studies to establish whether the functionalization occurs through the envisioned relay of the reactions (Fig. 2b) or through the direct $\mathrm{C}-\mathrm{H}$ bond activation of an alcohol via either $\mathrm{Ru}-$ or $\mathrm{Pd}$-complexes, potent catalysts for the $\mathrm{C}-\mathrm{H}$ bond functionalization. We confirmed the role of each catalyst and the reversible oxidation process. In the absence of the Pd-catalyst, no product 5a was formed (Supplementary Table 2). Likewise, without the $\mathrm{Ru}$-catalyst, the arylation of $1 \mathbf{a}$ was completely inhibited. In turn, 1-methoxyoctane - that is, the methyl ether derivative of 1-octanol 1a substrate that cannot be oxidized to the aldehyde intermediate - did not undergo the arylation. These results are consistent with the intermediacy of aldehydes $\mathbf{2}$ and $\mathbf{4}$ in the reaction. 
The further experimental data confirmed the hypothesized mechanism of the reaction shown in Fig. 2b. In general, the intermediates $\mathbf{2}$ and $\mathbf{4}$ are shuffled between the fast and reversible Ru-catalyzed activation cycle and the Pd-catalyzed arylation cycle, with the enolate formation as the ratedetermining step for the overall arylation of alcohol 1 to form product 5 (Fig. 3). Specifically, the kinetic experiments revealed that the reaction is positive order in both $\mathrm{Ru}$-complex and substrate $\mathbf{1}$, in agreement with the complex $[\mathrm{Ru}]$ and alcohol $\mathbf{1}$ being in the equilibrium with the hydride $[\mathrm{Ru}] \mathrm{H}_{2}$ and aldehyde $\mathbf{2}$. The activated intermediate $\mathbf{2}$ is then involved in the rate determining step (RDS) occurring in the arylation cycle operated by the Pd-catalyst. Accordingly, the reaction was found to be positive order in Pd-catalyst. Furthermore, the reaction is zero-order in aryl bromide (in the range of standard reaction concentrations), indicating that the oxidative-addition of aryl bromide is not rate-determining. In agreement, the reactions of alcohol 1 with a series of electron rich and electron deficient aryl bromides occurred at similar initial rates (Figure $3 b$ ). To identify the bond being broken during the RDS, we conducted the kinetic studies with selectively deuterium labeled alcohols $1 .{ }^{32}$ The side-byside kinetic experiments showed that the reactions for alcohols $\mathbf{1}$ containing deuterium labeling at the $\alpha-\mathrm{C}-\mathrm{H}$ and $\mathrm{O}-\mathrm{H}$ bonds, that is, the bonds that undergo oxidation in the presence of the Ru-catalyst, occurred at similar rates as the reaction for unlabeled alcohol $\mathbf{1}$ (Figure 3a). Considering that the Rucatalyzed oxidation reactions involving hydrogen transfer exhibit the clear primary kinetic isotope effects, ${ }^{33}$ and that the oxidation step is the first step in the sequence, prior to any deuterium scrambling possible, the lack of primary kinetic isotope effect here confirms that the oxidation is not involved in the RDS for the overall arylation reaction. In contrast, the reaction for the perdeuterated alcohol $\mathbf{1}$ occurs with 2-fold lower rate than the reaction for unlabeled alcohol 1 (Figure 3a), identifying that the reaction experiences the primary kinetic isotope effect. Considering no primary kinetic effect for the $\alpha-\mathrm{C}-\mathrm{H}$ and $\mathrm{O}-\mathrm{H}$ bonds, and the small influence of the secondary kinetic effects when compared with the primary kinetic effects, the observed overall isotope kinetic effect with the perdeuterated substrate is assigned primarily to the $\beta-\mathrm{C}-\mathrm{H}$ bond of alcohol $\mathbf{1}$, that is the bond that undergoes activation in the presence of the Pd-catalyst and the base. In agreement, the reaction for the alcohol $\mathbf{1}$ selectively deuterium labeled at the $\beta$-C-H bonds also occurs with 2-fold lower rate than the reaction for unlabeled alcohol 1 (Figure 3a). Overall, these data indicate the enolate formation as rate-limiting.

For comparison, the kinetic studies for the Pd-catalyzed arylation of aldehydes - that is, one part of the $\mathrm{Ru} / \mathrm{Pd}$-catalytic relay evaluated independently - indicated that the initial rate of the reaction exhibits strong dependence on the type of aryl bromide (Figure 3b). The reaction is zero-order in aldehyde and positive-order in aryl bromide, with the electron-rich aryl bromides reacting faster than the electron-deficient substrates (Figure 3b), indicating in that case the oxidative-addition as rate limiting. ${ }^{34,35}$ Furthermore, the kinetic experiments for non-deuterated and 2,2-bis-deuterated aldehydes 2 provided no primary KIE, with a value of 1.03(8), confirming that the enolate formation is non-rate 
limiting. Although the arylation of alcohols $\mathbf{1}$ and aldehydes $\mathbf{2}$ undergoes through the same sequence of the elementary steps occurring at the palladium center, the change of the rate limiting step most likely results from the significantly different regimes of concentrations of reactants and intermediates. For the direct arylation of aldehyde $\mathbf{2}$, the concentration of $\mathbf{2}$ is in large excess in respect to the concentration of the Pd-catalytic species. Therefore, the rate of the enolate formation is fast in respect to the rate of the oxidative-addition of aryl bromide, and the latter is rate limiting. In contrast, for the arylation of alcohol $\mathbf{1}$, the concentration of $\mathbf{2}$ is significantly lower, as it is determined by the equilibrium involving Ru-catalyst, which temporarily oxidizes only a small fraction of alcohol $\mathbf{1}$ to intermediate 2 . Therefore, in this case, the rate of the enolate formation becomes slow in respect to the rate of the oxidative-addition of aryl bromide, and the earlier is rate limiting. Overall, this comparison shows that the kinetic behavior of one reaction within a dual-catalytic system can differ from the kinetic behavior of the same reaction in a single-catalytic system. Thus, care needs to be taken when considering complex catalytic systems.

To get further insight, we also studied the possible cross-exchange processes. The arylation of a mixture of 1-butanol (1c) and perdeuterated alcohol 1c occurred with scrambling of deuterium in the $\alpha-\mathrm{C}-\mathrm{H}$ and $\beta-\mathrm{C}-\mathrm{H}$ positions of substrates early in the reaction (Figure 3c). This process further confirms that the reversible oxidation of alcohol $\mathbf{1}$ is faster than arylation of transient aldehyde $\mathbf{2}$. The reversible oxidation of $\mathbf{1}$ accounts for scrambling in the $\alpha-\mathrm{C}-\mathrm{H}$ positions. The reversible deprotonation of transient $\mathbf{2}$ accounts for scrambling in the $\beta-\mathrm{C}-\mathrm{H}$ positions. In turn, the reaction of perdeuterated 1c with bromobenzene (3a) in the presence non-deuterated $\beta$-aryl alcohol 5a led to deuterium incorporation into 5a (Figure 3c). This observation indicates that $\beta$-aryl alcohol product 5 remains in the equilibrium with transient aryl aldehyde $\mathbf{4}$ throughout the reaction. The potential role of the solvent in the hydrogen transfer was also considered. Under standard conditions, however, arylation of 1c conducted in a deuterated dioxane- $d_{8}$ formed $\beta$-aryl alcohol $\mathbf{5 c}$ without any deuterium incorporated (Figure 3d), excluding the solvent being involved in the hydrogen transfer. Overall, the body of experimental data is consistent with mode of cooperation between both orthogonal TM catalyzed reactions, as proposed in Fig. 2 b. 


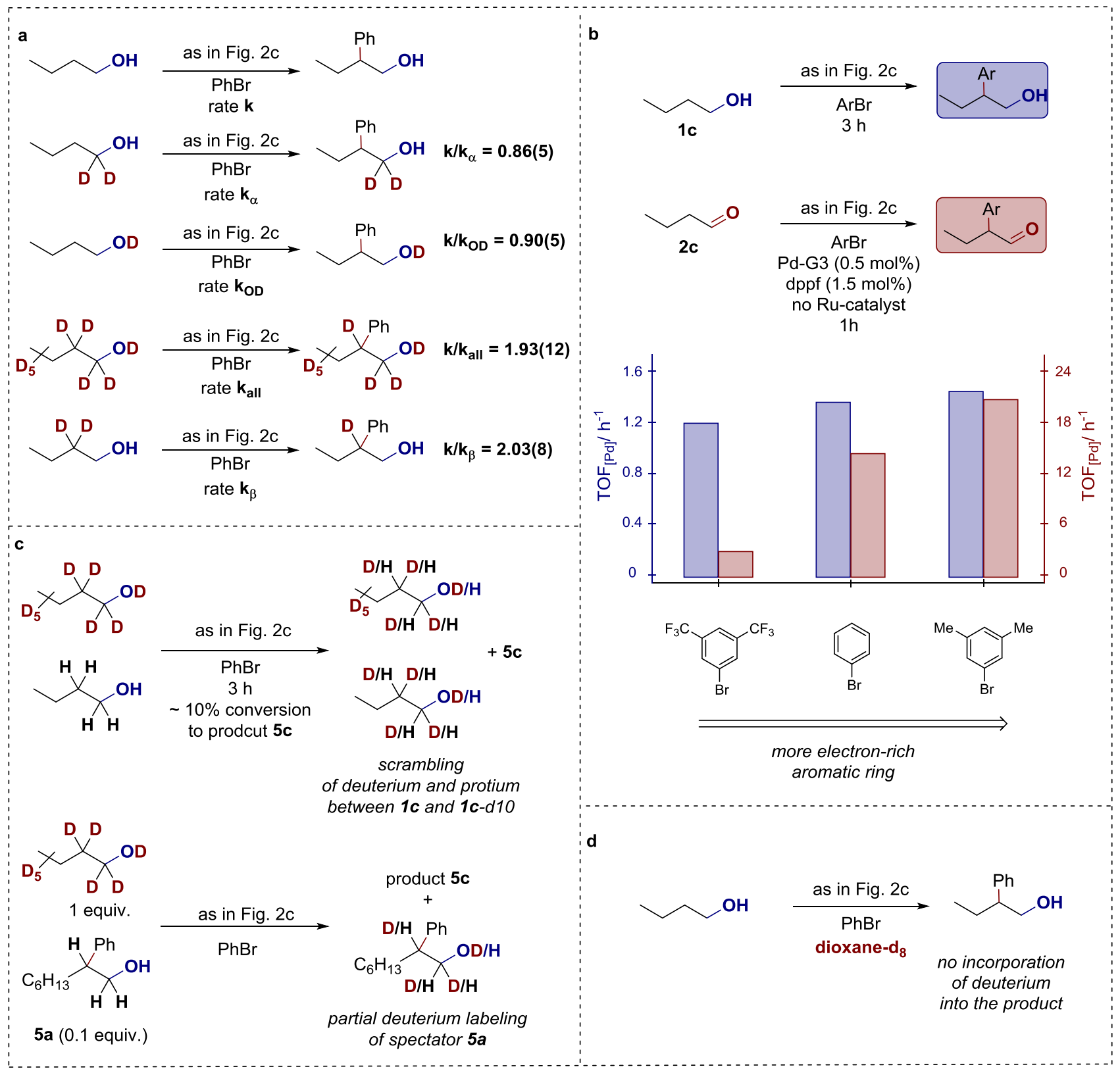

Figure 3 | Mechanistic studies. a, Studies of kinetic isotope effects (KIEs) supporting the scission of $\beta$-C-H bond occurring at the rate determining step for arylation of alcohols (the errors estimated as the standard deviations of the mean; no isotope distribution in the products were determined). $\mathbf{b}$, Initial rates for arylation of alcohol $\mathbf{1 c}$ and for arylation of aldehyde $\mathbf{2 c}$ with electronically varied aryl bromides, showing different influence of their electronic properties on the rate of the reaction. c, The experiments with deuterium labeled substrate showing the reversible oxidation of alcohols $\mathbf{1}$ and $\mathbf{5}$ to form aldehydes $\mathbf{2}$ and $\mathbf{4}$, respectively, which is fast in respect to the arylation step of $\mathbf{2}$ to form $\mathbf{4}$. d, Experiments with the deuterium labeled solvent excluding its active role in the hydrogen transfer. For the kinetic experiments and further details, see supplementary information.

Having established the $\mathrm{Ru}-/ \mathrm{Pd}$-catalyst relay, we evaluated the substrate scope of the reaction. We found that, under standard conditions, a range of aliphatic and aromatic alcohols $\mathbf{1 b}$-s containing various functional groups, including an alkene, amide, ester, alcohol and ketone, react to form $\beta$-aryl alcohols $\mathbf{5 b}$-s in up to $79 \%$ yields (Fig. 4a). Noteworthy, the alcohols $\mathbf{1 i - 1}$ containing either additional ketone or secondary alcohol moiety underwent arylation only at the $\beta-\mathrm{C}-\mathrm{H}$ position of the primary alcohol function (Fig. 4a). Although the secondary alcohol functions underwent oxidation under these 
conditions, no functionalization of the $\mathrm{C}-\mathrm{H}$ bonds neighboring the enolizable ketone or the secondary alcohol functions was observed, enabling chemoselective functionalization of poly-functionalized starting materials. The reactions of linear alcohols underwent to provide the $\beta$-aryl alcohols in the highest yields, however, $\beta$ - and $\gamma$-branched alcohols 1o-r containing sterically more encumbered $\beta$ $\mathrm{C}-\mathrm{H}$ bonds also reacted to form products 5o-5r in synthetically useful yields (38-60\%). In turn, the reactions of alcohols with a range of various aryl bromides delivered products $\mathbf{6 a - a c}$ in 40-86\% yields (Fig. 4b). The data demonstrate the tolerance of substitutions at every position of the aryl ring and of a variety of electron-withdrawing and electron-donating functional groups, such as alkyl, perfluoroalkyl, ether, thioether, chloride, nitrile, and ester groups. Also, aryl bromides with substituents other than phenyl rings, including heteroaromatics, such as naphthyl, pyridine, pyrimidine, quinoline and isoquinoline, readily reacted to form the $\beta$-aryl alcohols. In general, alcohols and (hetero)aryl bromides of different size and substitution pattern, and of different electronic properties containing various functional groups are competent substrates for this transformation. 


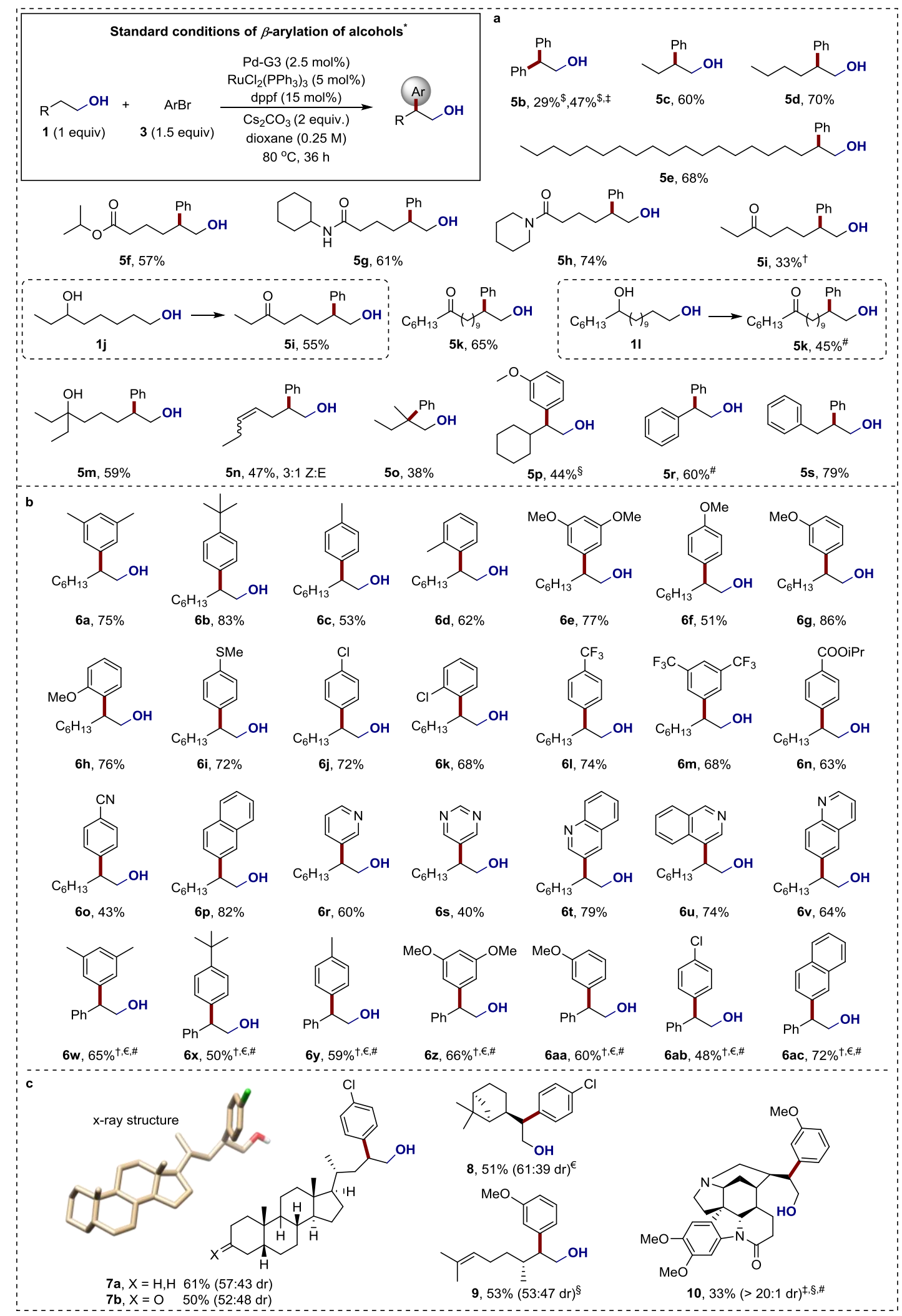

Figure 4 | Pd-/Ru-catalyzed arylation of $\boldsymbol{\beta}$-C-H bond of alcohols. a, Scope of alcohols. b, Scope of (hetero)aryl bromides. c, Reactions for natural product-like alcohols. *Reactions conducted on a $0.25-1 \mathrm{mmol}$ scale of alcohol using standard conditions: 0.5-1 equiv. of alcohol, 1.5 equiv. of $\mathrm{ArBr}, 2.5 \mathrm{~mol} \% \mathrm{Pd}-\mathrm{G} 3,5 \mathrm{~mol} \%$ $\mathrm{RuCl}_{2}\left(\mathrm{PPh}_{3}\right)_{3}, 15 \mathrm{~mol} \%$ dppf, 2 equiv. of $\mathrm{Cs}_{2} \mathrm{CO}_{3}, 4 \mathrm{ml}$ of dioxane for $1 \mathrm{mmol}$ of alcohol, $80{ }^{\circ} \mathrm{C}, 36 \mathrm{~h}$. Upon completion, the reaction was diluted with isopropanol $(4 \mathrm{ml})$ and incubated at $80{ }^{\circ} \mathrm{C}$ for $6 \mathrm{~h}$. $\mathrm{Ar}=\operatorname{aryl} ; \mathrm{Me}=$ methyl; $\mathrm{Ph}=$ phenyl. Yield (and $\mathrm{dr}=$ diastereomeric ratio) determined by ${ }^{1} \mathrm{H}$ NMR using an internal standard. Yields of isolated products using flash column chromatography with silica gel are usually lower of 5-10\%. \$Yield determined by GC using an internal standard. $\$ 0.5$ equivalent of alcohol. $†$ No incubation with isopropanol. $\$ 90{ }^{\circ} \mathrm{C}, 72 \mathrm{~h} . € 90{ }^{\circ} \mathrm{C}, 48 \mathrm{~h}$. \#Isolated yield. 
To further confirm the synthetic potential of the methodology, we explored the reactivity of more complexed alcohols (Fig. 4c). Under standard conditions, steroid (5 $\beta$ )-cholan-24-ol reacted with 4-bromochlorobenzene to form aryl product 7a in $61 \%$ yield, although with modest diastereoselectivity (57:43 dr). The reaction for a related substrate - a derivative of lithocholic acid - also occurred to form $\mathbf{7 b}$ in 50\% yield (52:48 dr), with no reactivity derived from its ketone moiety. Dihydronopol and (+)citronellol underwent the reactions to form 8 and $\mathbf{9}$, respectively, in 51\% yield (61:39 dr) and 53\% yield (53:47 dr). Finally, the derivative of alkaloid brucine, containing a number of functional groups - amine, amide, ether, and an electron-rich aromatic ring - underwent the reaction to form aryl product $\mathbf{1 0}$ as a single diastereomer in 33\% yield (>20:1 dr). The broad scope of substrates highlights the synthetic applicability of the methodology, which rests on its mild reagents and conditions.

To assess the generality of the approach further, we sought the dual-catalytic system for the direct $\gamma$-selective hydroarylation of allylic alcohols $\mathbf{1 1}$ that, like direct $\beta$-arylation of alcohols, has not been accomplished with single- or multicatalytic systems. ${ }^{36,37} \mathrm{We}$ envisioned that the combination of reversible oxidation of $\mathbf{1 1}$ with $\mathrm{Rh}$-catalyzed arylation ${ }^{38,39}$ of the corresponding transient unsaturated aldehyde 12 would afford the overall $\gamma$-selective hydroarylation (Fig. 5a). As a starting point, we evaluated an array of different $\mathrm{Ru}$-catalysts and $\mathrm{Rh}$-catalysts, for the hydroarylation of trans-2-hexen1-ol (11a) with 4-methoxyphenylboronic acid (15a). We found that 11a and 15a reacted selectively to form the aryl alcohol $(S)$-14a in $74 \%$ yield with a $96: 4$ enantiomeric ratio, in the presence of $\mathrm{RuH}_{2}\left(\mathrm{PPh}_{3}\right)_{4},\left[\mathrm{Rh}(\mathrm{cod})\left(\mathrm{CH}_{3} \mathrm{CN}\right)_{2}\right] \mathrm{BF}_{4}$, and $(R)$-BINAP (Fig. 5b). Although, the initial catalytic system offered limited scope of substrates, further experimentation using different hydrogen borrowing catalysts reveled that upon exchanging the $\mathrm{RuH}_{2}\left(\mathrm{PPh}_{3}\right)_{4}$ complex for the Fe-carbonyl complex developed by Knölker, ${ }^{40,41}$ the methodology is general and highly efficient for a broad spectrum of substrates (Fig. 5b). The reactions with a series of boronic acids and allylic alcohols occurred selectively to form the $\gamma$-aryl products with high enantiomeric ratios ranging 91:9 to 98:2 and in yields between $38-98 \%$ (Fig. 5b). In analogy to the methodology for the $\beta$-arylation of aliphatic alcohols described above, the methodology for $\gamma$-hydroarylation of allylic alcohols is compatible with a series of alcohols of different size and electronic properties, and it tolerates substitutions at every position of the aryl ring and a variety of electron-withdrawing and electron-donating functional groups, such as alkyl, perfluoroalkyl, ether, thioether, bromide, chloride, fluoride, N-Boc protected amine, and ester groups. Similarly, aryl boronic acids with substituents other than phenyl rings, including heteroaromatics, such as naphthyl, pyridine, N-protected and unprotected indole, benzofuran, (dibenzo)thiophene, all readily reacted to form the $\gamma$-aryl alcohol. In general, these examples highlight the great potential to enable new reactions with the TM dual-catalytic systems that exploit the catalytic reversible activation. 


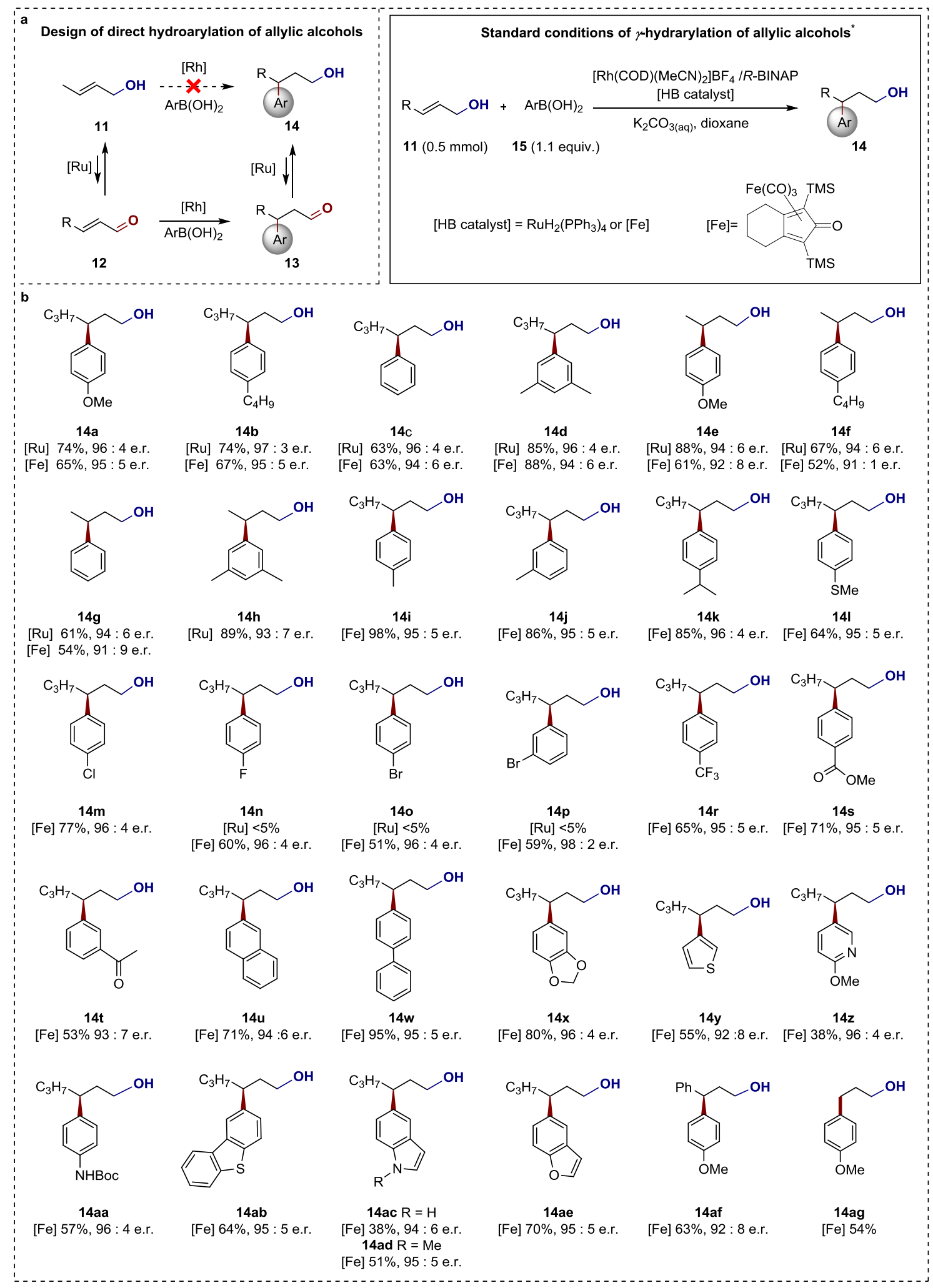

Figure $5 \mid \gamma$-Selective hydroarylation of allylic alcohols. a, Reaction design. b, Scope of substrates. *Reactions conducted on a $0.50 \mathrm{mmol}$ scale of alcohol using one set of standard conditions, noted as $[\mathrm{Ru}]$ or $[\mathrm{Fe}]$. $[\mathrm{Ru}]=$ 1.0 equiv. of alcohol, 1.1 equiv. of $\operatorname{ArB}(\mathrm{OH})_{2}, 2 \mathrm{~mol} \%\left[\mathrm{Rh}(\operatorname{cod})\left(\mathrm{CH}_{3} \mathrm{CN}\right)_{2}\right] \mathrm{BF}_{4}, 6 \mathrm{~mol} \% R$-BINAP, $10 \mathrm{~mol} \%$ $\mathrm{RuH}_{2}\left(\mathrm{PPh}_{3}\right)_{4}, 2$ equiv. of $\mathrm{K}_{2} \mathrm{CO}_{3}$ as $0.2 \mathrm{ml}$ aqueous solution, $1 \mathrm{ml}$ of dioxane, $50{ }^{\circ} \mathrm{C}, 30 \mathrm{~h}$. [Fe] = 1.0 equiv. of alcohol, 1.1 equiv. of $\mathrm{ArB}(\mathrm{OH})_{2}, 2 \mathrm{~mol} \%$ [Rh(cod) $\left.\left(\mathrm{CH}_{3} \mathrm{CN}\right)_{2}\right] \mathrm{BF}_{4}, 3$ mol\% R-BINAP, 7.5 mol\% Knölker Fecomplex, ${ }^{42} 11.25 \mathrm{~mol} \%$, trimethylamine N-oxide, 1 equiv. of $\mathrm{K}_{2} \mathrm{CO}_{3}$ as $0.1 \mathrm{ml}$ aqueous solutions, $1 \mathrm{ml}$ of dioxane, $90{ }^{\circ} \mathrm{C}, 48 \mathrm{~h}$. Upon completion, the reactions were diluted with isopropanol $(1 \mathrm{ml})$ and incubated at 80 ${ }^{\circ} \mathrm{C}$ for $1 \mathrm{~h}$. Yield determined by ${ }^{1} \mathrm{H}$ NMR using an internal standard. Yields of isolated products using flash column chromatography with silica gel are usually lower of 5-10\%. Enantiomeric ratio (e. r.) was determined by chiral HPLC analysis. 


\section{Conclusions}

In summary, we report dual-catalytic systems that merge TM catalyzed reversible activation reactions and TM catalyzed functionalization reactions to undergo transformations of substrates at their unreactive sites, which are otherwise directly inaccessible. Conveniently, these systems engage known catalytic cycles for the activation and for the functionalization cycles, accelerating the development of these new transformations. We envision that by exploiting different combinations of TM catalyzed activation and TM catalyzed functionalization cycles, following the principles of the straightforward reaction design, such dual-catalytic systems will open a new realm of possible transformations for substrates at their otherwise unreactive sites, voiding the need for the use of highly reactive reagents or the multi-step procedures. We believe that the study will facilitate the development of transformations, which are currently unattainable but are desired for both the development and production of fine-chemicals and materials.

Supporting Information: All experimental details, supplemental figures and tables, and spectra are available online in the supporting information file.

Data availability: The authors declare that the data supporting the findings of this study are available within the paper and its supplementary information files.

Acknowledgments: This work was financially supported by the University of Strasbourg, the French National Research Agency ('Investments for the future' program of the IdEx Unistra framework), FRC \& LabEx Chemistry of Complex Systems, the Polish National Science Centre (Etiuda fellowship no. 2016/20/T/ST5/00494 to DL), the European Union (Marie Curie Actions, PCOFUND-GA-2013609102) through the Campus France (Prestige fellowship no. PRESTIGE-2017-4-0022 to DL), the Polish Ministry of Science and Higher Education (Mobilnosc Plus fellowship no. 1672/1/MOB/V/1 7/2018/0 to KH), and the Foundation for Polish Science (Start fellowship no. START- 036.2018 to $\mathrm{KH})$. We thank Dr. L. Karmazin for the crystallographic measurements, Dr. E. Richmond for help with the initial HPLC analysis, and Dr. W. Dzik for helpful discussions.

\section{Author Contributions}

DL and PD conceived, designed and performed the initial experiments. DL, YZ and PD designed and performed subsequent experiments. DL, YZ and KH perform the experiments during the revision. PD conceived the concept and prepared the manuscript with feedback from DL. 


\section{Author Information and Affiliation}

${ }^{1}$ University of Strasbourg, CNRS, ISIS UMR 7006, F-67000 Strasbourg, France.

*Corresponding author (dydio@unistra.fr)

\section{References and Notes}

1. Hartwig, J. F. Organotransition metal chemistry: from bonding to catalysis. (University Science Books, 2010).

2. Hartwig, J. F. \& Larsen, M. A. Undirected, homogeneous C-H bond functionalization: challenges and opportunities. ACS Cent. Sci. 2, 281-292 (2016).

3. Sambiagio, C. et al. A comprehensive overview of directing groups applied in metal-catalysed C-H functionalisation chemistry. Chem. Soc. Rev. 47, 6603-6743 (2018).

4. Cernak, T., Dykstra, K. D., Tyagarajan, S., Vachal, P. \& Krska, S. W. The medicinal chemist's toolbox for late stage functionalization of drug-like molecules. Chem. Soc. Rev. 45, 546-576 (2016).

5. Blakemore, D. C. et al. Organic synthesis provides opportunities to transform drug discovery. Nat. Chem. 10, 383-394 (2018).

6. Gandeepan, P. \& Ackermann, L. Transient directing groups for transformative C-H activation by synergistic metal catalysis. Chem 4, 199-222 (2018).

7. Zhang, F.-L., Hong, K., Li, T.-J., Park, H. \& Yu, J.-Q. Functionalization of C(sp3)-H bonds using a transient directing group. Science 351, 252-256 (2016).

8. Park, H., Verma, P., Hong, K. \& Yu, J.-Q. Controlling Pd(iv) reductive elimination pathways enables Pd(ii)-catalysed enantioselective $\mathrm{C}\left(\mathrm{sp}^{3}\right)-\mathrm{H}$ fluorination. Nat. Chem. 10, 755-762 (2018).

9. Corma, A., Navas, J. \& Sabater, M. J. Advances in one-pot synthesis through borrowing hydrogen catalysis. Chem. Rev. 118, 1410-1459 (2018).

10. Watson, A. J. A. \& Williams, J. M. J. The give and take of alcohol activation. Science 329, 635$636(2010)$.

11. Wang, D. \& Astruc, D. The golden age of transfer hydrogenation. Chem. Rev. 115, 6621-6686 (2015). 
12. Hill, C. K. \& Hartwig, J. F. Site-selective oxidation, amination and epimerization reactions of complex polyols enabled by transfer hydrogenation. Nat. Chem. 9, 1213-1221 (2017).

13. Guillena, G., Ramón, D. J. \& Yus, M. Hydrogen autotransfer in the $N$-alkylation of amines and related compounds using alcohols and amines as electrophiles. Chem. Rev. 110, 1611-1641 (2010).

14. Bähn, S. et al. The catalytic amination of alcohols. ChemCatChem 3, 1853-1864 (2011).

15. Yang, Q., Wang, Q. \& Yu, Z. Substitution of alcohols by N-nucleophiles via transition metalcatalyzed dehydrogenation. Chem. Soc. Rev. 44, 2305-2329 (2015).

16. Obora, Y. Recent advances in $\alpha$-alkylation reactions using alcohols with hydrogen borrowing methodologies. ACS Catal. 4, 3972-3981 (2014).

17. Gunanathan, C. \& Milstein, D. Applications of acceptorless dehydrogenation and related transformations in chemical synthesis. Science 341, 1229712-1229712 (2013).

18. Bender, M., Turnbull, B. W. H., Ambler, B. R. \& Krische, M. J. Ruthenium-catalyzed insertion of adjacent diol carbon atoms into C-C bonds: Entry to type II polyketides. Science 357, 779-781 (2017).

19. Nguyen, K. D. et al. Metal-catalyzed reductive coupling of olefin-derived nucleophiles: Reinventing carbonyl addition. Science 354, aah5133 (2016).

20. Black, P. J., Harris, W. \& Williams, J. M. J. Catalytic electronic activation: indirect addition of nucleophiles to an allylic alcohol. Angew. Chem. Int. Ed. 40, 4475 (2001).

21. Quintard, A., Constantieux, T. \& Rodriguez, J. An iron/amine-catalyzed cascade process for the enantioselective functionalization of allylic alcohols. Angew. Chem. Int. Ed. 52, 12883-12887 (2013).

22. Roudier, M., Constantieux, T., Quintard, A. \& Rodriguez, J. Triple iron/copper/iminium activation for the efficient redox neutral catalytic enantioselective functionalization of allylic alcohols. ACS Catal. 6, 5236-5244 (2016).

23. Goldman, A. S., Roy, A. H., Huang, Z., Schinski, W. \& Brookhart, M. Catalytic alkane metathesis by tandem alkane dehydrogenation-olefin metathesis. Science 312, 257-261 (2006). 
24. Haibach, M. C., Kundu, S., Brookhart, M. \& Goldman, A. S. Alkane metathesis by tandem alkane-dehydrogenation-olefin-metathesis catalysis and related chemistry. Acc. Chem. Res. 45, 947-958 (2012).

25. Mo, F., Tabor, J. R. \& Dong, G. Alcohols or masked alcohols as directing groups for C-H bond functionalization. Chem. Lett. 43, 264-271 (2014).

26. Bellina, F. \& Rossi, R. Transition metal-catalyzed direct arylation of substrates with activated sp 3 -hybridized $\mathrm{C}-\mathrm{H}$ bonds and some of their synthetic equivalents with aryl halides and pseudohalides. Chem. Rev. 110, 1082-1146 (2010).

27. Smith, A. M. R. \& Hii, K. K. (Mimi). Transition metal catalyzed enantioselective $\alpha$ heterofunctionalization of carbonyl compounds. Chem. Rev. 111, 1637-1656 (2011).

28. Pirnot, M. T., Rankic, D. A., Martin, D. B. C. \& MacMillan, D. W. C. Photoredox activation for the direct beta-arylation of ketones and aldehydes. Science 339, 1593-1596 (2013).

29. Terrett, J. A., Clift, M. D. \& MacMillan, D. W. C. Direct $\beta$-alkylation of aldehydes via photoredox organocatalysis. J. Am. Chem. Soc. 136, 6858-6861 (2014).

30. Zhang, X. \& MacMillan, D. W. C. Direct aldehyde C-H arylation and alkylation via the combination of nickel, hydrogen atom transfer, and photoredox catalysis. J. Am. Chem. Soc. 139, 11353-11356 (2017).

31. Hazari, N., Melvin, P. R. \& Beromi, M. M. Well-defined nickel and palladium precatalysts for cross-coupling. Nat. Rev. Chem. 1, 0025 (2017).

32. Simmons, E. M. \& Hartwig, J. F. On the interpretation of deuterium kinetic isotope effects in C$\mathrm{H}$ bond functionalizations by transition-metal complexes. Angew. Chem. Int. Ed. 51, 3066-3072 (2012).

33. Samec, J. S. M., Bäckvall, J.-E., Andersson, P. G. \& Brandt, P. Mechanistic aspects of transition metal-catalyzed hydrogen transfer reactions. Chem. Soc. Rev. 35, 237 (2006).

34. Alcazar-Roman, L. M. \& Hartwig, J. F. Mechanistic studies on oxidative addition of aryl halides and triflates to $\mathrm{Pd}(\mathrm{BINAP}) 2$ and structural characterization of the product from aryl triflate addition in the presence of amine. Organometallics 21, 491-502 (2002). 
35. Hartwig, J. F. Electronic effects on reductive elimination to form carbon-carbon and carbonheteroatom bonds from palladium(11) complexes. Inorg. Chem. 46, 1936-1947 (2007).

36. Werner, E. W., Mei, T.-S., Burckle, A. J. \& Sigman, M. S. Enantioselective Heck arylations of acyclic alkenyl alcohols using a redox-relay strategy. Science 338, 1455-1458 (2012).

37. Mei, T.-S., Patel, H. H. \& Sigman, M. S. Enantioselective construction of remote quaternary stereocentres. Nature 508, 340-344 (2014).

38. Tian, P., Dong, H.-Q. \& Lin, G.-Q. Rhodium-catalyzed asymmetric arylation. ACS Catal. 2, 95119 (2012).

39. Hayashi, T. \& Yamasaki, K. Rhodium-catalyzed asymmetric 1,4-addition and its related asymmetric reactions. Chem. Rev. 103, 2829-2844 (2003).

40. Bauer, I. \& Knölker, H.-J. Iron catalysis in organic synthesis. Chem. Rev. 115, 3170-3387 (2015).

41. Bullock, R. M. An iron catalyst for ketone hydrogenations under mild conditions. Angew. Chem. Int. Ed. 46, 7360-7363 (2007).

42. Knölker, H.-J., Baum, E., Goesmann, H. \& Klauss, R. Demetalation of tricarbonyl(cyclopentadienone)iron complexes initiated by a ligand exchange reaction with $\mathrm{NaOH}-\mathrm{X}$-Ray analysis of a complex with nearly square-planar coordinated sodium. Angew. Chem. Int. Ed. 38, 2064-2066 (1999). 